\title{
Tumor Necrosis Factor-Like Weak Inducer of Apoptosis Increases the Permeability of the Neurovascular Unit through Nuclear Factor- $\kappa$ B Pathway Activation
}

\author{
Rohini Polavarapu, ${ }^{1}$ Maria Carolina Gongora, ${ }^{1}$ Jeffrey A. Winkles, ${ }^{2}$ and Manuel Yepes ${ }^{1}$ \\ ${ }^{1}$ Department of Neurology and Center for Neurodegenerative Disease, Emory University School of Medicine, Atlanta, Georgia 30322, and ${ }^{2}$ Departments of \\ Surgery and Physiology and the Center for Vascular and Inflammatory Diseases, University of Maryland School of Medicine, Baltimore, Maryland 21201
}

\begin{abstract}
Tumor necrosis factor-like weak inducer of apoptosis (TWEAK) is a member of the tumor necrosis factor superfamily. TWEAK acts on responsive cells via binding to a small cell-surface receptor named fibroblast growth factor-inducible-14 (Fn14). TWEAK can stimulate numerous cellular responses including cell proliferation, migration, and proinflammatory molecule production. The present study investigated whether TWEAK plays a role in the regulation of the permeability of the neurovascular unit (NVU). We found that intracerebral injection of TWEAK in wild-type mice induces activation of the nuclear factor- $\kappa \mathrm{B}(\mathrm{NF}-\kappa \mathrm{B})$ pathway and matrix metalloproteinase-9 (MMP-9) expression in the brain with resultant disruption in the structure of the NVU and increase in the permeability of the blood- brain barrier (BBB). TWEAK did not increase MMP-9 activity or BBB permeability when injected into mice genetically deficient in the NF- $\kappa$ B family member 550 . Furthermore, we report that inhibition of TWEAK activity during cerebral ischemia with an Fn14-Fc decoy receptor results in significant preservation of the integrity of the NVU with attenuation of cerebral ischemia-induced increase in the permeability of the BBB. We conclude that the cytokine TWEAK plays a role in the disruption of the structure and permeability of the NVU during physiological and pathological conditions.
\end{abstract}

Key words: TWEAK; NF- $\kappa$ B; neurovascular unit; blood- brain barrier; matrix metalloproteinase-9; cerebral ischemia

\section{Introduction}

Tumor necrosis factor (TNF)-like weak inducer of apoptosis (TWEAK) is a member of the TNF superfamily of cytokines (Chicheportiche et al., 1997). TWEAK is synthesized as a type II transmembrane protein, but it can be cleaved to generate a soluble factor with biological activity (Chicheportiche et al., 1997). TWEAK activity is mediated via binding to fibroblast growth factor-inducible-14 (Fn14), a member of the TNF receptor superfamily (Wiley et al., 2001). TWEAK binding to Fn14 activates the nuclear factor $-\kappa \mathrm{B}(\mathrm{NF}-\kappa \mathrm{B})$ pathway (Donohue et al., 2003; Han et al., 2003; Saitoh et al., 2003; Jin et al., 2004; Kim et al., 2004; Xu et al., 2004; Tran et al., 2005). TWEAK has been reported to stimulate cell proliferation (Lynch et al., 1999; Harada et al., 2002; Donohue et al., 2003), migration (Harada et al., 2002; Donohue et al., 2003; Tran et al., 2003), and differentiation (Polek et al., 2003), as well as the expression of proinflammatory molecules (Chicheportiche et al., 1997, 2002; Saas et al., 2000; Harada et al., 2002; Jin et al., 2004; Kim et al., 2004; Xu et al.,

Received June 14, 2005; revised Sept. 19, 2005; accepted Sept. 23, 2005.

This work was supported in part by National Institutes of Health Grants HL-39727 (J.A.W.) and NS-49478 (M.Y.). We thank Hong Yi from the Emory University School of Medicine Electron Microscopy Core Facility for her assistance with the electron microscopy studies.

Correspondence should be addressed to Manuel Yepes, Department of Neurology and Center for Neurodegenerative Disease, Emory University School of Medicine, Whitehead Biomedical Research Building, 615 Michael Street, Suite 505J, Atlanta, GA 30322. E-mail: myepes@emory.edu.

DOI:10.1523/JNEUROSCI.3382-05.2005

Copyright $\odot 2005$ Society for Neuroscience $\quad$ 0270-6474/05/2510094-07\$15.00/0
2004). TWEAK is also an angiogenic factor (Lynch et al., 1999; Ho et al., 2004), and it has been shown that TWEAK plays a role in the pathophysiology of cerebral ischemia (Potrovita et al., 2004; Yepes et al., 2005).

The neurovascular unit (NVU) consists of endothelial cells, the basal lamina, the astrocytic foot processes, pericytes, and neurons. The NVU forms a barrier, known as the blood-brain barrier $(\mathrm{BBB})$, which regulates the entry of selected molecules from the blood into the CNS (Rubin and Staddon, 1999; Ballabh et al., 2004). In pathologic situations such as acute cerebral ischemia and head trauma, increases in BBB permeability lead to vasogenic edema (Baker et al., 1971; Garcia et al., 1978). Several proinflammatory cytokines including TNF- $\alpha$, interleukin-1 (IL-1), IL-6, IL- 8 , and interferon- $\lambda$ have been implicated in the regulation of BBB permeability (Matsumoto et al., 1997; Bolton et al., 1998; Mayhan, 2002).

The NF- $\kappa \mathrm{B} /$ reticuloendotheliosis (Rel) family of transcription factors have also been implicated in increased BBB permeability and cell death (Xu et al., 2002; Nadjar et al., 2003). These factors control the transcription of genes involved in the immune response, inflammation, apoptosis, and tumorigenesis (Pahl, 1999; Lin and Karin, 2003; Chen and Greene, 2004; Shishodia and Aggarwal, 2004). NF- $\kappa \mathrm{B}$ is expressed at low levels in the CNS (Kaltschmidt et al., 1994). However, a number of events including seizures (Rong and Baudry, 1996) and cerebral ischemia (Schneider et al., 1999) induce activation of the NF- $\kappa$ B pathway in the brain. The matrix metalloproteinase-9 (MMP-9) gene is an 
NF- $\kappa$ B-regulated gene (Bond et al., 1998), and MMP-9 has a direct effect on the permeability of the NVU in several pathological conditions (Romanic and Madri, 1994; Rosenberg et al., 1996; Asahi et al., 2000, 2001). In the studies presented here, we demonstrate that TWEAK stimulates MMP-9 activity and disrupts the architecture of the NVU and that these effects depend on activation of the NF- $\kappa \mathrm{B}$ signaling pathway.

\section{Materials and Methods}

Animal models. For the analysis of BBB permeability, either C57BL/6J wild-type mice or C57/Sv129 NF- $\kappa$ B p50 $0^{-1-}$ knock-out mice and their littermate wild-type controls (The Jackson Laboratory, Bar Harbor, ME) were used. All procedures were approved by the Emory University Institutional Animal Care and Use Committee. Mice were anesthetized with a mixture of ketamine and xylazine, placed on a stereotactic frame, and injected with $2 \mu \mathrm{l}$ of either PBS or TWEAK $(1 \mu \mathrm{g} / \mu \mathrm{l}$; R \& D Systems, Minneapolis, MN) in the left striatum, at the following coordinates: bregma, -2; mediolateral, 1; and dorsoventral, 3 (Paxinos and Franklin, 2001). For the animal model of cerebral ischemia, the middle cerebral artery was exposed and occluded with a 10-0 suture as described previously (Nagai et al., 1999). Cerebral perfusion (CP) in the distribution of the middle cerebral artery was monitored throughout the surgical procedure with a laser Doppler (Perimed, North Royalton, $\mathrm{OH}$ ), and only animals with a $>80 \%$ decrease in $\mathrm{CP}$ were included in this study. The rectal and masseter muscle temperatures were controlled at $37^{\circ} \mathrm{C}$ with a homeothermic blanket. Immediately after middle cerebral artery occlusion (MCAO), animals were placed on a stereotactic frame, and $2 \mu \mathrm{l}$ of PBS, Fn14-Fc decoy $(1 \mu \mathrm{g} / \mu \mathrm{l})$, or Fc protein $(1 \mu \mathrm{g} / \mu \mathrm{l})$, purified as described previously (Donohue et al., 2003; Yepes et al., 2005), was injected into the third ventricle at the following coordinates: bregma, -2 ; mediolateral, 0; dorsoventral, 2. Seventy-two hours later, brains were removed, and the permeability of the NVU was measured as described below.

Analysis of the permeability of the NVU. Immediately after the intracerebral injection of TWEAK or PBS or the intraventricular injection of PBS, Fn14-Fc decoy, or Fc, animals were injected intravenously with $2 \mu \mathrm{l}$ of $2 \%$ Evans blue dye (Sigma, St. Louis, MO). After 24 or $72 \mathrm{~h}$, animals were perfused transcardially with PBS, brains were removed, weighed, homogenized in $400 \mu \mathrm{l}$ of $N, N$-dimethylformamide (Sigma), and centrifuged at $21,000 \times g$ for $30 \mathrm{~min}$. Evans blue was quantified from the absorbance at $620 \mathrm{~nm}$ of each supernatant minus the background calculated from the baseline absorbance between 500 and $740 \mathrm{~nm}$ and divided by the wet weight of each hemisphere. To see whether the effect of TWEAK on BBB permeability was mediated by TNF- $\alpha, 15 \mu \mathrm{g}$ of antimouse TNF- $\alpha$ antibodies (R \& D Systems) was injected into the third ventricle in a subgroup of animals followed $30 \mathrm{~min}$ later by the intracerebral injection of TWEAK and the intravenous administration of Evans blue dye. Animals were killed $24 \mathrm{~h}$ later, and Evans blue dye extravasation was quantitated as described above.

Immunogold electron microscopy. Mice were anesthetized deeply $24 \mathrm{~h}$ after the intracerebral injection of TWEAK or PBS or the intraventricular injection of PBS or Fn14-Fc. Brains were fixed by transcardial perfusion with $4 \%$ paraformaldehyde and $0.1 \%$ glutaraldehyde in $0.1 \mathrm{~m}$ phosphate buffer, $\mathrm{pH} 7.2$, for 20 min followed by immersion-fixation with $2 \%$ paraformaldehyde in the same buffer overnight. Fifty micrometer cuts were obtained with a vibrating microtome, fixed with $0.5 \%$ osmium tetroxide for $15 \mathrm{~min}$, dehydrated, and embedded in Eponate 12 resin. After the polymerization of the resin, areas of interests were dissected out from flat-embedded vibrating microtome sections and re-embedded. Sixty nanometer cuts were obtained, stained with uranyl acetate and lead citrate, and examined with a Hitachi (Tokyo, Japan) H-7500 transmission electron microscope.

Western blot analysis. Polyclonal antibodies to phospho-inhibitor of $\kappa \mathrm{B} \alpha(\mathrm{I} \kappa \mathrm{B} \alpha)$, total $\mathrm{I} \kappa \mathrm{B} \alpha$, and $\mathrm{p} 100 / 52$ were purchased from Cell Signaling Technology (Beverly, MA). Antibodies to $\beta$-actin were obtained from Sigma. Animals were injected with $2 \mu \mathrm{l}(1 \mu \mathrm{g} / \mathrm{ul})$ of TWEAK or PBS at the coordinates described above, and brains were extracted at $0,1,3$, or $6 \mathrm{~h}$ and homogenized in lysis buffer containing $2 \%$ SDS, $0.25 \mathrm{M}$ Tris- $\mathrm{HCl}$, and $1 \%$ protease inhibitor mixture (Roche, Indianapolis, IN). Samples were then centrifuged at $12,000 \times g$ for $15 \mathrm{~min}$, and protein concentrations were determined using the BCA protein assay (Bio-Rad, Hercules, CA). Equal amounts of protein were loaded on a $12 \%$ polyacrylamide gel and transferred onto a polyvinylidene difluoride membrane. Blots were blocked with 5\% nonfat milk in TBS and incubated in a 1:1000 dilution of the primary antibody overnight at $4^{\circ} \mathrm{C}$. The blots were then incubated with IR Dye 800-conjugated secondary antibodies (Rockland, Gilbersville, PA), and the signal was read with a LI-COR Biosciences (Lincoln, $\mathrm{NE}$ ) Odyssey blot imaging system. Each experiment was repeated four times. The mean density of the band was measured directly from the gel using the NIH Image analyzer system, and statistical analysis was performed using Student's $t$ test.

Immunofluorescence. Mice were perfused transcardially with PBS $1 \mathrm{~h}$ after the intracerebral injection of TWEAK. Frozen brains were cut in 10 $\mu \mathrm{m}$ sections, immersed in $100 \%$ methanol for $10 \mathrm{~min}$, incubated in citrate buffer for $10 \mathrm{~min}$, washed three times in PBS, blocked with 5\% goat serum for $1 \mathrm{~h}$, and incubated with an anti-p65 antibody (Santa Cruz Biotechnology, Santa Cruz, CA) and a rabbit anti-glial fibrillary acidic protein (GFAP) antibody (DakoCytomation, Carpinteria, CA), neuronspecific nuclear protein (NeuN) monoclonal antibody (Chemicon, Temecula, CA), or purified anti-mouse CD11b (MAC1) monoclonal antibody (eBioscience, San Diego, CA) for $1 \mathrm{~h}$ at room temperature. After three washes with PBS, a 1:500 dilution of goat anti-rabbit secondary antibody conjugated to Alexa 488 (Invitrogen, San Diego, CA) or donkey anti-mouse or anti-goat secondary antibodies conjugated to Rhodamine Red-X (Jackson ImmunoResearch, West Grove, PA) were diluted to 1:500 and applied for $1 \mathrm{~h}$ at room temperature. Finally, the sections were counterstained with 4,6-diamidino-2-phenylindole (Invitrogen) for 2-3 s. Coverslips were rinsed with PBS and mounted on glass slides with Gel/Mount (Biomedia, Foster City, CA).

MMP-9 activity analysis. For analysis of MMP-9 activity in murine brains, animals were killed 6,12 , or $24 \mathrm{~h}$ after the intracerebral injection of TWEAK or PBS. The brains were removed, divided into ipsilateral and contralateral hemispheres, and homogenized in a 2:1 v/w ratio of lysis buffer containing $0.5 \mathrm{M} \mathrm{NaCl}, 1 \%$ Triton X-100 (Sigma), $0.05 \%$ Tween 20 (Sigma), and $50 \mathrm{~mm}$ sodium acetate. The samples were centrifuged at $10,000 \times g$ for $10 \mathrm{~min}$, and the supernatant was added to Gelatin Sepharose $4 \mathrm{~B}$ beads (Amersham Biosciences, Piscataway, NJ). After $3 \mathrm{~h}$ incubation at room temperature, the beads were washed three times in PBS, incubated in sample buffer for $30 \mathrm{~min}$, and centrifuged. The supernatant was then loaded in a gelatin zymography gel (Invitrogen). After electrophoresis, the gel was washed twice during 45 min with $2.5 \%$ Triton X-100 (Sigma), followed by a $30 \mathrm{~min}$ wash with Tris-buffered saline containing $5 \mathrm{mM} \mathrm{CaCl}_{2}$, then incubated overnight at $37^{\circ} \mathrm{C}$ in the same buffer, after which the gel was stained with $0.5 \%$ Coomassie brilliant blue R-250 (Amersham Biosciences) for $30 \mathrm{~min}$ and then destained. Purified murine MMP-9 from Chemicon was used as a marker for MMP-9. For analysis of MMP-9 activity in cultured cells, astrocytes were prepared from postnatal day 1 mice as described previously (Yepes et al., 2005). Cells were cultured in a serum-free media and incubated with TWEAK $(100 \mathrm{ng} / \mathrm{ml})$ for $24 \mathrm{~h}$. MMP-9 activity was measured in the lysate and the media as described above. Each experiment was performed four times, and the density of the band in each observation was analyzed using the $\mathrm{NIH}$ Image analyzer system. Statistical analysis was performed with Student's $t$ test.

\section{Results}

\section{TWEAK increases the permeability of the}

\section{blood-brain barrier}

To study the effect of TWEAK on the permeability of the BBB, the cytokine was injected directly into the mouse brain, and the extravasation of Evans blue dye was quantified by spectrofluorophotometric analysis. We found that the intracerebral injection of TWEAK results in a dose-dependent increase in the permeability of the BBB (Fig. 1). The recombinant TWEAK protein used in these experiments was purified from bacteria. To confirm that TWEAK and not contaminating bacterial endotoxin (lipopolysaccharide) was responsible for the observed effect, we incubated 

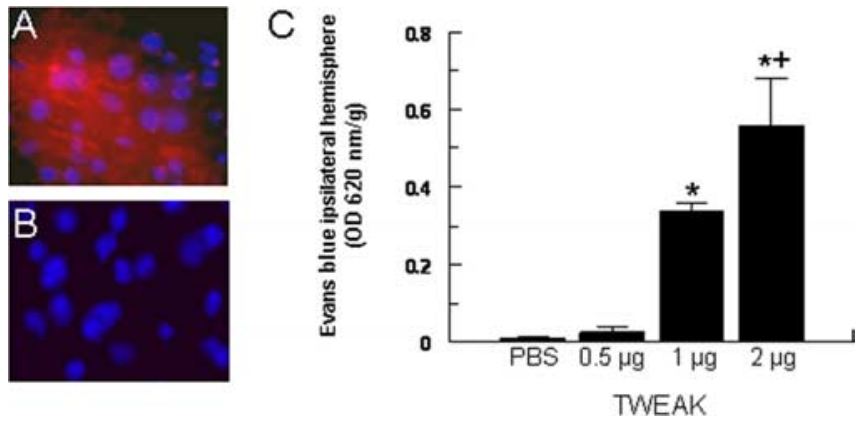

Figure 1. TWEAK induces a dose-dependent increase in BBB permeability. $A, B$, Evans blue dye extravasation after the intracerebral injection of $2 \mu \mathrm{g}$ of TWEAK $(\boldsymbol{A})$ or PBS $(\boldsymbol{B})$. Blue is $4^{\prime}, 6^{\prime}$-diamidino-2-phenylindole, and red is Evans blue dye. Magnification, 100X. C, Quantification of Evans blue dye extravasation in response to intracerebral injection of either PBS or TWEAK. Bars denote mean value $(n=6-10)$, and error bars describe SEM. ${ }^{*} p<0.0001$ compared with PBS and $0.5 \mu \mathrm{g} ;{ }^{+} p=0.0005$ compared with $1 \mu \mathrm{g}$.

the TWEAK protein at $95^{\circ} \mathrm{C}$ for $10 \mathrm{~min}$ before the intracerebral injection. No effect on BBB permeability was observed after the intracerebral injection of heat-inactivated TWEAK (data not shown). Likewise, no effect on TWEAK-induced increase in BBB permeability was observed in animals pretreated with antiTNF- $\alpha$ antibodies, demonstrating that the effect of TWEAK on $\mathrm{BBB}$ permeability is not an indirect effect mediated by the NF$\kappa \mathrm{B}$-regulated cytokine TNF- $\alpha$ (data not shown).

TWEAK disrupts the architecture of the neurovascular unit We then investigated whether the observed effect of TWEAK on the permeability of the $\mathrm{BBB}$ was attributable to changes in the architecture of the NVU. In these experiments, either PBS or TWEAK was injected into the brain, and tissue was harvested $24 \mathrm{~h}$ later. The architecture of the NVU was evaluated by immunogold electron microscopy. We found that the intracerebral injection of TWEAK results in the accumulation of fluid in the perivascular space (Fig. $2 B$ ). This effect is associated with disruption of the glia limitans and detachment of the astrocytic foot processes with development of perivascular edema (Fig. 2D). These observations demonstrate that the effect of TWEAK on the permeability of the $\mathrm{BBB}$ is associated with structural changes in the architecture of the NVU.

\section{Effect of TWEAK on NF- $\kappa$ B pathway activation in vivo}

Activation of the NF- $\kappa \mathrm{B}$ pathway has been implicated in changes in the permeability of the $\mathrm{BBB}$ in response to treatment with cytokines (Nadjar et al., 2003) and exposure to hypoxic conditions in vitro (Brown et al., 2003; Witt et al., 2005). NF- $\kappa$ B complexes can be activated via two distinct pathways (Lin and Karin, 2003; Chen and Greene, 2004; Shishodia and Aggarwal, 2004). In the classic or "canonical" pathway, NF- $\kappa \mathrm{B}$ complexes are present in the cytoplasm as inactive factors as a consequence of their association with the repressor protein $\mathrm{I} \kappa \mathrm{B} \alpha$. Stimulation of cells induces $\mathrm{I} \kappa \mathrm{B} \alpha$ phosphorylation and proteosome-mediated degradation. This results in the liberation of NF- $\kappa \mathrm{B}$ [usually p50/p65 $($ RelA)], allowing nuclear translocation and binding to DNA. In the alternative or "noncanonical" pathway, $\mathrm{I} \kappa \mathrm{B} \alpha$ is not involved but instead extracellular stimuli induce proteolytic processing of NF- $\kappa$ B2 (p100), which promotes nuclear translocation of $\mathrm{p} 52$ homodimers or $\mathrm{p} 52 /$ RelB heterodimers.

To determine whether TWEAK could stimulate the NF- $\kappa \mathrm{B}$ pathway in vivo, we first analyzed $\mathrm{I} \kappa \mathrm{B} \alpha$ phosphorylation and p100 processing, indicators of activation of the canonical and
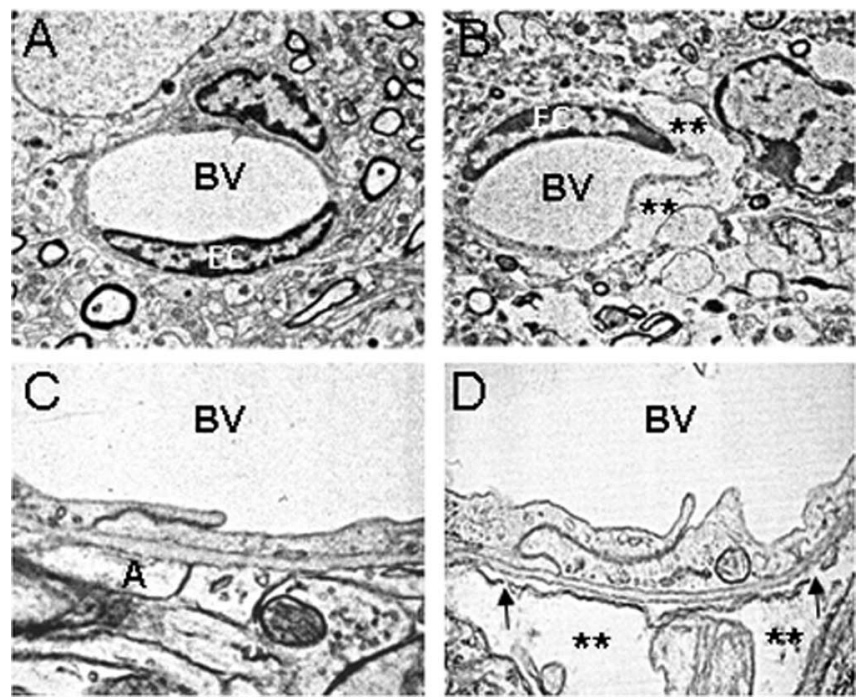

Figure 2. TWEAK disrupts the structure of the NVU. Electron microscopy of cerebral arterioles in the left striatum of mouse brains injected with either $\operatorname{PBS}(\boldsymbol{A}, \boldsymbol{C})$ or TWEAK $(\boldsymbol{B}, \boldsymbol{D})$ is shown. The asterisks show fluid-filled spaces indicative of developing edema in the TWEAK-treated brain $(\boldsymbol{B}, \boldsymbol{D})$. The arrows indicate places in the neurovascular unit with disruption of the glia limitans and detachment of the astrocytic processes. BV, Blood vessel; $A$, astrocytic processes. Magnification: $A, B, 5000 \times ; C, D, 30,000 \times$.
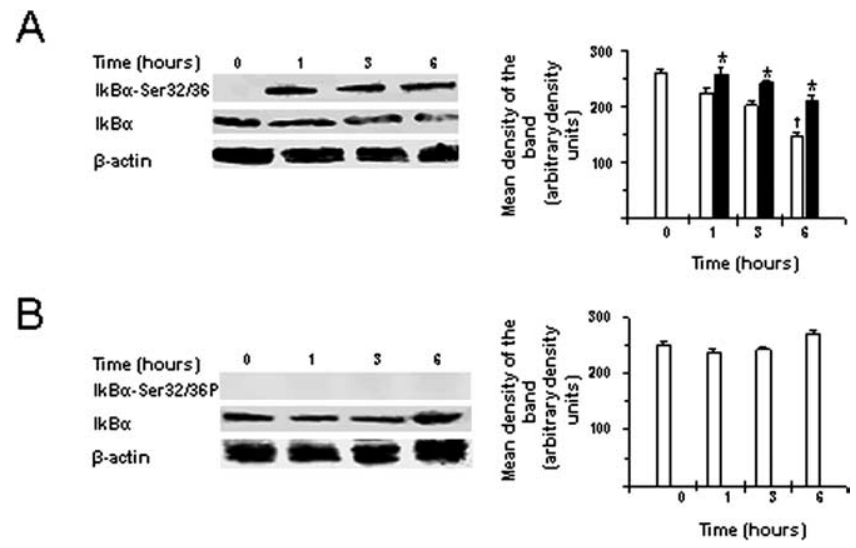

Figure 3. TWEAK stimulates NF- $\kappa$ B activation in the brain. Western blot analysis of $I_{\kappa} \mathrm{B} \alpha$ phosphorylation and total $I \kappa \mathrm{B} \alpha$ expression in brain extracts $0,1,3$, and $6 \mathrm{~h}$ after the intracerebral injection of either TWEAK $(\boldsymbol{A})$ or PBS $(\boldsymbol{B})$ is shown. $n=4$ for each observation. Bars denote mean intensity of the band corresponding to total $\kappa_{\kappa} B \alpha$ levels (white bars) or phospho$\mathrm{I}_{\kappa \mathrm{B}} \alpha$ levels (black bars). Error bars describe SEM. ${ }^{*} p<0.02$ for phospho- $\mathrm{I}_{\kappa} \mathrm{B} \alpha$ levels, and ${ }^{\dagger} p<0.02$ for total $l_{\kappa} B \alpha$ levels compared with corresponding levels at time 0 . Actin expression levels were also assayed as a control for protein loading.

noncanonical NF- $\kappa$ B pathways, respectively, at $0,1,3$, or $6 \mathrm{~h}$ after the intracerebral injection of the cytokine. Brain lysates were prepared and Western blot analysis performed using appropriate antibodies. We detected $\mathrm{I} \kappa \mathrm{B} \alpha$ phosphorylation as early as $1 \mathrm{~h}$ after treatment with TWEAK with a progressive decrease of total $\mathrm{I} \kappa \mathrm{B} \alpha$ levels (Fig. $3 A$ ). No effect on $\mathrm{I} \kappa \mathrm{B} \alpha$ phosphorylation or total $\mathrm{I} \kappa \mathrm{B} \alpha$ levels was observed in vehicle-injected brains (Fig. 3B). Neither TWEAK nor vehicle injection stimulated p100 processing to the p52 subunit (data not shown).

To study what cell types in the brain were responding to TWEAK administration, we injected TWEAK, harvested the brain tissue $1 \mathrm{~h}$ later, and performed immunofluorescence analysis using antibodies that recognize neurons, microglia, or astrocytes in combination with an anti-p65 antibody. The p50/ 


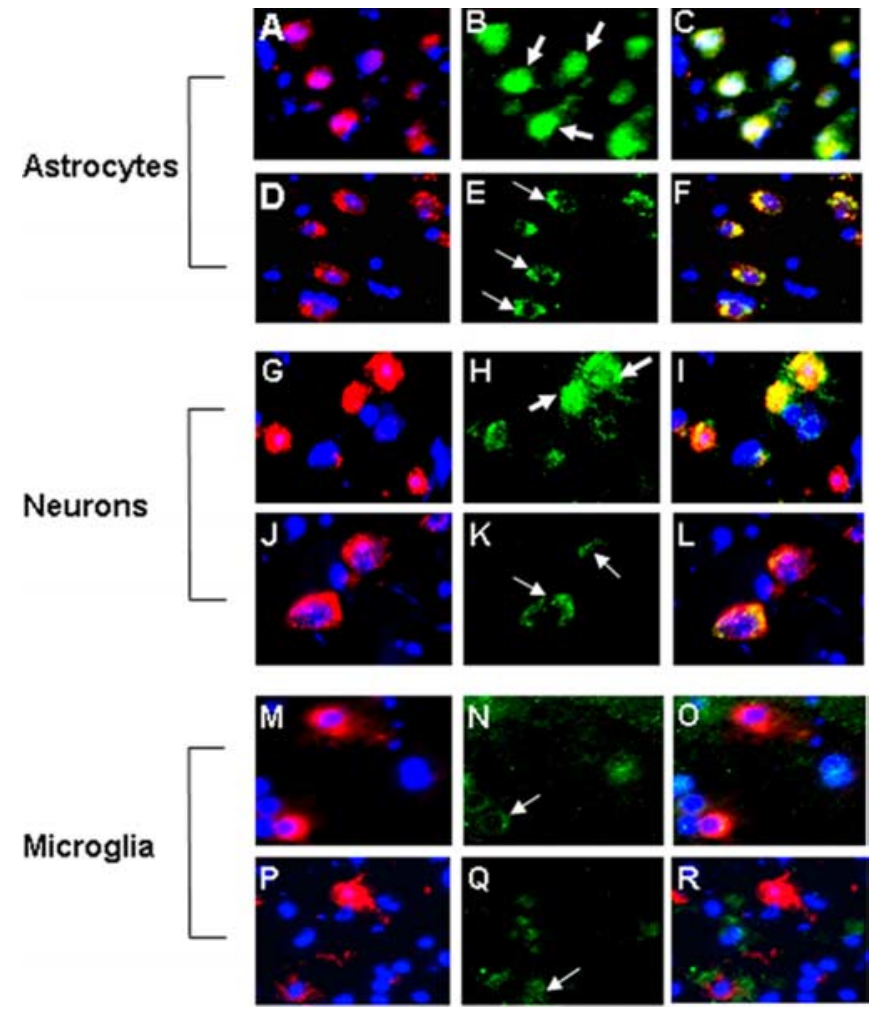

Figure 4. Effect of TWEAK on NF- $\kappa B$ pathway activation in astrocytes, neurons, and microglia. Indirect immunofluorescence analysis of p65 subcellular location in murine astrocytes $(\boldsymbol{A}-\boldsymbol{F})$, neurons $(\boldsymbol{G}-\boldsymbol{L})$, and microglia $(\boldsymbol{M}-\boldsymbol{R}), 1 \mathrm{~h}$ after the intracerebral injection of TWEAK, is shown. Frozen sections of brains injected with TWEAK $(\boldsymbol{A}-\boldsymbol{C}, \mathbf{G}-\mathbf{I}, \boldsymbol{M}-\mathbf{0})$ or PBS (D-F, $\boldsymbol{J}-\boldsymbol{L}, \boldsymbol{P}-\boldsymbol{R})$ are shown. Red is GFAP staining in $\boldsymbol{A}$ and $\boldsymbol{D}$, neuronal (NeuN) staining in $\boldsymbol{G}$ and $\boldsymbol{J}$, and MAC-1 staining in $\boldsymbol{M}$ and $\boldsymbol{P}$. Blue is $4^{\prime}, \boldsymbol{6}^{\prime}$-diamidino-2-phenylindole. Green is p65 staining in $\boldsymbol{B}, \boldsymbol{E}, \boldsymbol{H}, \boldsymbol{K}$, $\boldsymbol{M}, \boldsymbol{N}$, and $\boldsymbol{Q} . \boldsymbol{C}, \boldsymbol{F}, \boldsymbol{I}, \boldsymbol{L}, \boldsymbol{O}$, and $\boldsymbol{R}$ represent the corresponding merged images. Thick arrows indicate cells with nuclear translocation of the $\mathrm{p} 65$ subunit. Thin arrows indicate the cytoplasmic location of the $p 65$ subunit of the inactive NF- $\kappa$ B complex. Magnification, $100 \times$.

p65(RelA) NF- $\kappa$ B complex is normally retained in the cytoplasm as a result of its association with $\mathrm{I} \kappa \mathrm{B} \alpha$, but after $\mathrm{I} \kappa \mathrm{B} \alpha$ phosphorylation and degradation, this complex enters the nucleus. Thus, p65 nuclear translocation is an indicator of canonical NF- $\kappa \mathrm{B}$ pathway activation. We detected TWEAK-induced translocation of the p65 subunit from the cytoplasm into the nucleus in the vast majority of astrocytes and in some neurons but in very few microglial cells (Fig. 4).

\section{Effect of TWEAK on MMP-9 activation}

MMP-9 plays an important role in the proteolytic degradation of the NVU (Asahi et al., 2000, 2001). To determine whether TWEAK can regulate MMP-9 activity in vivo, murine brains were injected with either TWEAK or PBS, tissue was harvested at 6, 12, or $24 \mathrm{~h}$, and MMP-9 activity was analyzed by gelatin zymography. We observed a progressive increase in MMP-9 activity in mice treated with TWEAK (Fig. 5A, lanes 2-4) but not with PBS (Fig. $5 A$, lanes 5-7).

Because the p65 immunofluorescence studies demonstrated that TWEAK was primarily acting on astrocytes in vivo (Fig. 4), we determined whether TWEAK treatment of murine astrocytes cultured in vitro increased MMP-9 activity. We found a significant increase in MMP-9 activity in both the lysate and the media in TWEAK-treated astrocytes (Fig. 6, lanes 2 and 4). In contrast, no MMP-9 activity was observed in PBS-treated cultures (Fig. 6, lanes 3 and 5). These results demonstrate that TWEAK induces
A

$\mathrm{B}$
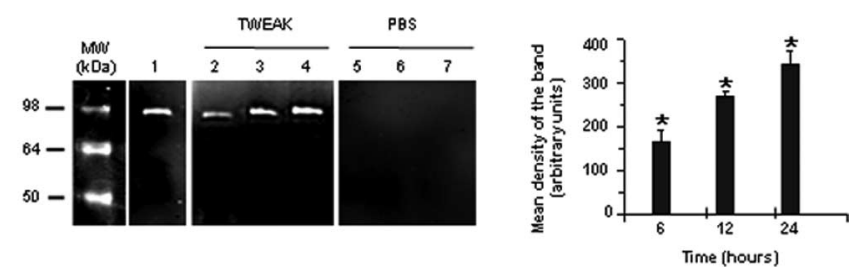

Figure 5. TWEAK increases MMP-9 activity in the brain. A, Gelatin zymography assay. Lane 1 is purified murine MMP-9, and all other lanes show MMP-9 activity after injection of either TWEAK (lanes 2-4) or PBS (lanes 5-7). Brains were extracted at $6 \mathrm{~h}$ (lanes 2 and 5), $12 \mathrm{~h}$ (lanes 3 and 6), or $24 \mathrm{~h}$ (lanes 4 and 7) after the intracerebral injections and gelatin zymography was performed. $\boldsymbol{B}$, Quantification of the mean density of the bands $(n=4)$. Bars represent the mean density of the bands in TWEAK- (black bars) or PBS- (white bars) treated brains. Error bars represent SEM. ${ }^{*} p<0.005$ compared with PBS injected brains. MW, Molecular weight.

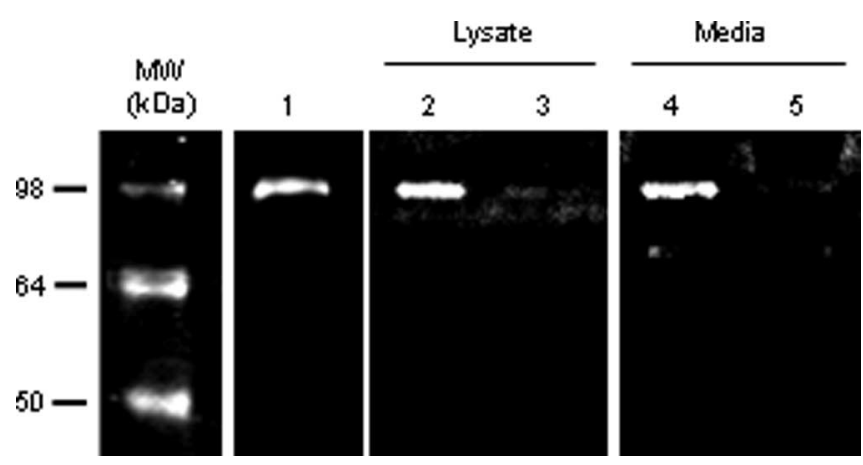

Figure 6. TWEAK treatment of murine astrocytes increases MMP-9 activity. Gelatin zymography analysis. Lane 1 is purified murine MMP-9, and all other lanes show MMP-9 activity after astrocyte exposure to either TWEAK (lanes 2 and 4) or PBS (lanes 3 and 5). Lanes 2 and 3 represent MMP- 9 activity in cell lysates, and lanes 4 and 5 depict MMP- 9 activity in the corresponding media. MW, Molecular weight.

MMP-9 activation in astrocytes and suggest that this activation may be responsible for the observed effect of TWEAK on the structure of the NVU and the permeability of the BBB.

The TWEAK-induced increase in MMP-9 activation and BBB permeability is mediated by the canonical NF- $\kappa \mathrm{B}$ pathway

To determine whether TWEAK-induced MMP-9 activation was mediated through the canonical NF- $\kappa$ B pathway, MMP-9 activity was analyzed $24 \mathrm{~h}$ after the intracerebral injection of TWEAK or PBS in wild-type and $\mathrm{p} 50$-deficient $\left(\mathrm{p} 50^{-1-}\right)$ mice. We found an increase in MMP-9 activity in wild-type animals treated with TWEAK (Fig. 7A, lanes 2 and 3 ). In contrast, no MMP-9 activity was found in either wild-type mice treated with PBS (Fig. 7A, lanes 4 and 5) or in p50 $0^{-/-}$animals treated with TWEAK or PBS (Fig. 7A, lanes 6-9).

To study whether the observed increase in BBB permeability after treatment with TWEAK was also dependent on NF- $\kappa \mathrm{B}$ pathway activation, we quantified the extravasation of Evans blue dye in wild-type and $\mathrm{p}^{-1-}$ mice $24 \mathrm{~h}$ after the intracerebral injection of TWEAK. We observed an increase in BBB permeability after the injection of TWEAK in wild-type animals. In contrast, this effect was significantly decreased in $\mathrm{p} 50^{-1-}$ mice (Fig. $7 C)$. Together, these data demonstrate that TWEAK induces MMP-9 activation in vivo through NF- $\kappa \mathrm{B}$ and suggest that this $\mathrm{NF}-\kappa \mathrm{B}$-mediated activation of MMP- 9 may result in degradation of the NVU. 

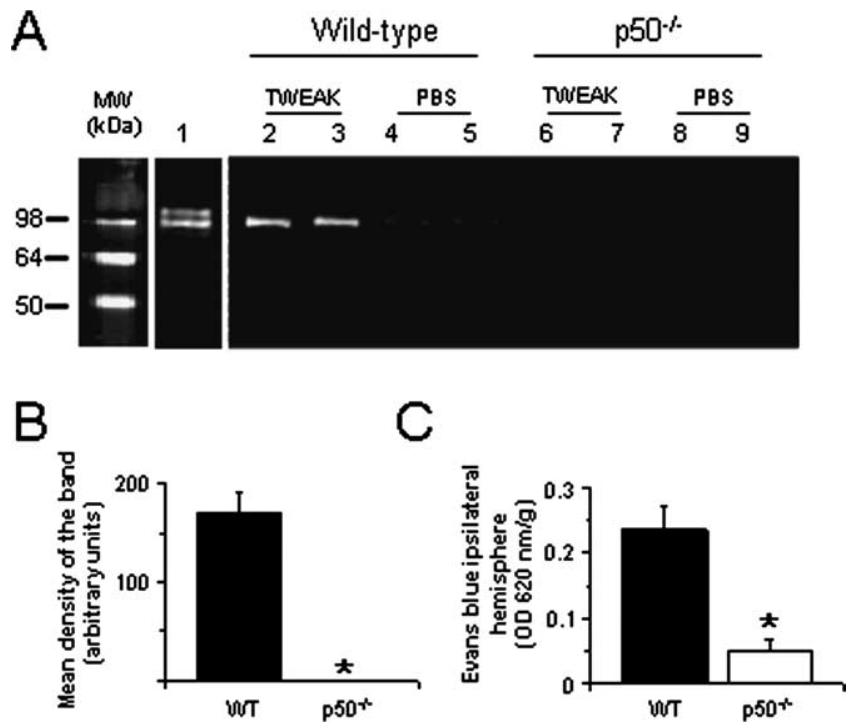

Figure 7. The NF- $\kappa B$ signaling pathway mediates TWEAK-induced MMP-9 activation and opening of the BBB. $\boldsymbol{A}$, Gelatin zymography of brains treated with either TWEAK or PBS. Lane 1 is purified murine MMP-9. Lanes 2-5 show MMP-9 activity in wild-type mice injected with either TWEAK (lanes 2 and 3) or PBS (lanes 4 and 5). Lanes 6 -9 show MMP-9 activity in p50 - mice injected with either TWEAK (lanes 6 and 7) or PBS (lanes 8 and 9). Brains were harvested $24 \mathrm{~h}$ after the injection of TWEAK or PBS. $\boldsymbol{B}$, Mean intensity of the band in wild-type and p50 ${ }^{-1-}$ mice injected with TWEAK $(n=4) .{ }^{*} p<0.005$ compared with wild-type mice. $C$, Quantification of Evans blue dye extravasation $24 \mathrm{~h}$ after the intracerebral injection of TWEAK in wild-type (WT) and p50-deficient animals. Bars denote mean value $(n=6)$, and error bars describe SEM. * $p<0.005$ compared with wild-type mice. MW, Molecular weight.

Effect of endogenously expressed TWEAK on the structure of the NVU and the permeability of the BBB

In previous experiments, we demonstrated that after the onset of cerebral ischemia, there is an increase in TWEAK expression in the area of ischemic penumbra and that neutralization of this TWEAK with a soluble Fn14-Fc decoy receptor results in a decrease in the volume of the ischemic lesion (Yepes et al., 2005). To examine whether the TWEAK expressed during cerebral ischemia has an effect on the structure of the NVU and the permeability of the BBB, wild-type mice underwent MCAO followed by the intraventricular injection of PBS, Fn14-Fc, or Fc. Evans blue dye extravasation was analyzed $72 \mathrm{~h}$ later. We found that inhibition of endogenous TWEAK activity with the Fn14-Fc decoy results in a significant decrease of ischemia-induced increase in BBB permeability (Fig. $8 A, B$ ). To further characterize this observation, we performed immunogold electron microscopy of murine brains after MCAO and treatment with either PBS or Fn14Fc. We observed that after MCAO and treatment with PBS, there is accumulation of fluid in the perivascular space as a result of increase in the permeability of the NVU (Fig. $8 C$, top). In contrast, in animals in which the endogenous activity of TWEAK was inhibited by treatment with Fn14-Fc, there was preservation in the architecture of the NVU with significant attenuation of the development of perivascular edema (Fig. 8C, bottom).

\section{Discussion}

The NVU regulates the supply of nutrients to the CNS and prevents the passage of harmful substances from the intravascular space into the brain (Zonta et al., 2003; Leybaert, 2005). Disruption of the NVU occurs in a number of pathological conditions including cerebral ischemia, head trauma, CNS infections, and multiple sclerosis (Garcia et al., 1978; Hawkins et al., 1991; Banks, 1999; Yepes et al., 2003; Ballabh et al., 2004) and results in the development of cerebral edema, which is a frequent cause of mortality in these patients. Thus, understanding the pathophysiological processes leading to disruption of the NVU and increase in BBB permeability is crucial for the development of therapeutic strategies for these conditions.

Our results demonstrate that TWEAK induces a dosedependent increase in the permeability of the NVU. Furthermore, electron microscopy analysis revealed that treatment with TWEAK results in disruption of the glia limitans, detachment of astrocytic foot processes, and development of perivascular edema. These changes are associated with neuronal death in the neighboring areas. The TWEAK-induced increase in BBB permeability may indirectly cause cell death in these areas. However, it has been reported that exposure of primary murine neuronal cultures to recombinant TWEAK under oxygen-glucose deprivation conditions results in apoptotic cell death (Potrovita et al., 2004). Thus, it is also possible that TWEAK may directly stimulate neuronal death in vivo.

Previous studies have shown that TWEAK and Fn14 are expressed by astrocytes in vivo (Yepes et al., 2005) and that in vitro exposure of astrocytes to TWEAK results in the release of IL-6 and IL-8 (Saas et al., 2000; Kim et al., 2004). Thus, it is possible that the injection of TWEAK in our model results in the release of proinflammatory cytokines from astrocytes and that the interaction of these molecules with the NVU results in increase in the permeability of the BBB. TWEAK could also be having an effect on the NVU via binding to Fn14 receptors on brain endothelial cells (Wiley et al., 2001; Harada et al., 2002; Donohue et al., 2003).

Activation of NF- $\kappa \mathrm{B}$ has been described in astrocytes in several neuropathological processes such as cerebral ischemia (Schneider et al., 1999; Zhang et al., 2005), head trauma (Nonaka et al., 1999), and seizures (Yu et al., 1999). It has been shown that TWEAK binding to its receptor Fn14 results in activation of the NF- $\kappa \mathrm{B}$ pathway in vitro (Donohue et al., 2003; Han et al., 2003; Saitoh et al., 2003; Jin et al., 2004; Kim et al., 2004; Xu et al., 2004; Tran et al., 2005). To study whether TWEAK can activate NF- $\kappa$ B in vivo, we injected TWEAK directly into the brain of wild-type mice and evaluated NF- $\kappa \mathrm{B}$ activation by immunoblotting and immunofluorescence. Our results demonstrate that TWEAK induces a rapid activation of the canonical NF- $\kappa \mathrm{B}$ pathway in vivo and that this effect seems to occur primarily in astrocytes.

$\mathrm{NF}-\kappa \mathrm{B}$ activates genes that are known to increase the permeability of the NVU (Bond et al., 1998; Nadjar et al., 2003). Indeed, it has been demonstrated that MMP-9 activity is regulated through the NF- $\kappa$ B pathway (Bond et al., 1998) and that this metalloproteinase has a direct effect on the permeability of the NVU (Asahi et al., 2000, 2001; Aoki et al., 2002). Furthermore, in vitro studies have shown that TWEAK can induce MMP-9 activity in macrophages and Eph4 cells (Kim et al., 2004; Michaelson et al., 2005). To study whether TWEAK has an effect in vivo on MMP-9 activity in the CNS, we performed gelatin zymographic assays in murine brains injected with TWEAK. We found an increase in MMP-9 activity as early as $6 \mathrm{~h}$ after the intracerebral injection of TWEAK. Furthermore, TWEAK treatment of astrocytes cultured in vitro also increased MMP-9 activity.

To examine directly the role of NF- $\kappa \mathrm{B}$ activation in the observed TWEAK effects, we injected TWEAK protein into the brains of $\mathrm{p} 50^{-1-}$ mice. Our results demonstrate that in contrast to wild-type animals, treatment with TWEAK does not result in an increase in MMP-9 activity in $\mathrm{p}^{-1-}$ mice, providing further support to our hypothesis that TWEAK induces MMP-9 activity in vivo through activation of the canonical NF- $\kappa \mathrm{B}$ pathway. To see whether this effect of TWEAK on NF- $\kappa$ B and MMP-9 has an 
A

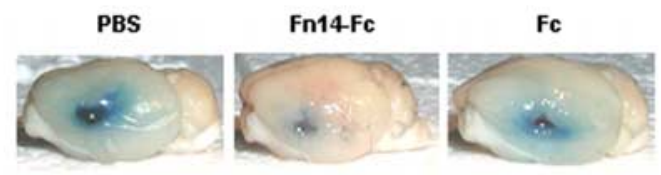

B

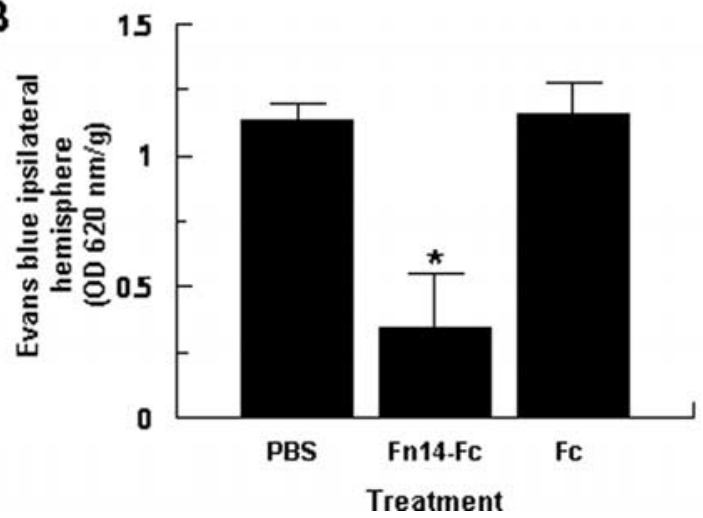

C

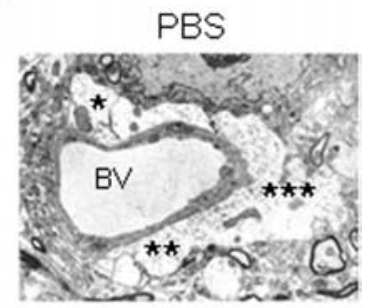

$\mathrm{Fn} 14-\mathrm{Fc}$

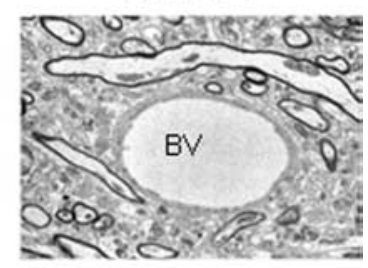

signal during stroke, there is release of TWEAK from astrocytes. This TWEAK binds to the Fn14 receptor, also on astrocytes, resulting in activation of the NF- $\kappa \mathrm{B}$ pathway and induction of proinflammatory cytokines and MMP-9 with resultant disruption of the structure of the NVU and increase in BBB permeability. Inhibition of TWEAK with Fn14-Fc decoy results in protection of the NVU and preservation of the integrity of the BBB.

\section{References}

Aoki T, Sumii T, Mori T, Wang X, Lo EH (2002) Blood-brain barrier disruption and matrix metalloproteinase-9 expression during reperfusion injury: mechanical versus embolic focal ischemia in spontaneously hypertensive rats. Stroke 33:2711-2717.

Asahi M, Asahi K, Jung JC, del Zoppo GJ, Fini ME, Lo EH (2000) Role for matrix metalloproteinase 9 after focal cerebral ischemia: effects of gene knockout and enzyme inhibition with BB-94. J Cereb Blood Flow Metab 20: $1681-1689$.

gigure 8. Effect of endogenous TWEAK on the permeability of the BBB and the integrity of the NVU. $A$, Evans blue dye extravasation $72 \mathrm{~h}$ after $\mathrm{MCAO}$ and the intraventricular injection of $\mathrm{PBS}, \mathrm{Fn} 14-\mathrm{Fc}$, or $\mathrm{Fc}$. The blue staining denotes the areas with increased BBB permeability. B, Quantification of Evans blue dye extravasation after MCA0 and treatment with PBS, Fn14-Fc, or Fc. Error bars represent SEM. ${ }^{*} p<0.05$ compared with PBS- or Fc-treated brains $(n=4)$. C, Electron microscopy of cerebral arterioles of mouse brains subjected to MCAO and intraventricular administration of either PBS (top) or Fn14-Fc decoy (bottom). The asterisks show fluid-filled spaces indicative of developing edema in the PBS-treated brain. BV, Blood vessel. Magnification, $5000 \times$.

effect on BBB permeability, we evaluated the extravasation of Evans blue dye in $\mathrm{p} 0^{-1-}$ mice injected with TWEAK. We found that in contrast to wild-type animals, the intracerebral injection of TWEAK in $\mathrm{p}^{50^{-1-}}$ mice resulted in a minimal increase in BBB permeability. Together, our results demonstrate that the intracerebral injection of TWEAK results in augmentation in the permeability of the NVU by activation of the NF- $\kappa$ B pathway and resultant increase in MMP-9 activity.

Finally, we also investigated whether endogenously expressed TWEAK could regulate the permeability of the NVU. The onset of cerebral ischemia is followed by increase in the permeability of the NVU with development of vasogenic edema (Garcia et al., 1978). In previous studies, we demonstrated that cerebral ischemia was accompanied by an increase in TWEAK and Fn14 expression in the area surrounding the necrotic core (Yepes et al., 2005). Also, inhibition of this endogenous TWEAK with an Fn14-Fc decoy receptor resulted in decrease of the volume of the ischemic lesion (Yepes et al., 2005). To see whether the protective effect of Fn14-Fc decoy on cerebral ischemia was attributable to inhibition of the deleterious effects of TWEAK on the structure of the NVU, mice underwent MCAO followed by the intraventricular injection of PBS, Fn14-Fc decoy, or Fc. Brains were extracted $72 \mathrm{~h}$ later, and Evans blue dye extravasation was analyzed. We found that TWEAK inhibition resulted in a significant decrease in ischemia-induced increase in BBB permeability. Electron microscopy analysis showed areas of perivascular edema in the PBStreated animals. In contrast, this area of edema was significantly attenuated in Fn14-Fc decoy-treated mice, demonstrating that during cerebral ischemia endogenous TWEAK induces changes in the architecture of the NVU with resultant increase in the permeability of the BBB.

In summary, our results demonstrate that TWEAK has a direct effect on the structure of the NVU and the permeability of the BBB. We postulate a model in which in response to the ischemic
Asahi M, Wang X, Mori T, Sumii T, Jung JC, Moskowitz MA, Fini ME, Lo EH (2001) Effects of matrix metalloproteinase- 9 gene knock-out on the proteolysis of blood-brain barrier and white matter components after cerebral ischemia. J Neurosci 21:7724-7732.

Baker RN, Cancilla PA, Pollock PS, Frommes SP (1971) The movement of exogenous protein in experimental cerebral edema. An electron microscopic study after freeze-injury. J Neuropathol Exp Neurol 30:668-679.

Ballabh P, Braun A, Nedergaard M (2004) The blood-brain barrier: an overview: structure, regulation, and clinical implications. Neurobiol Dis 16:1-13.

Banks WA (1999) Physiology and pathology of the blood-brain barrier: implications for microbial pathogenesis, drug delivery and neurodegenerative disorders. J Neurovirol 5:538-555.

Bolton SJ, Anthony DC, Perry VH (1998) Loss of the tight junction proteins occludin and zonula occludens-1 from cerebral vascular endothelium during neutrophil-induced blood-brain barrier breakdown in vivo. Neuroscience $86: 1245-1257$.

Bond M, Fabunmi RP, Baker AH, Newby AC (1998) Synergistic upregulation of metalloproteinase- 9 by growth factors and inflammatory cytokines: an absolute requirement for transcription factor NF-kappa B. FEBS Lett 435:29-34.

Brown RC, Mark KS, Egleton RD, Huber JD, Burroughs AR, Davis TP (2003) Protection against hypoxia-induced increase in blood-brain barrier permeability: role of tight junction proteins and NF-kappaB. J Cell Sci 116:693-700.

Chen LF, Greene WC (2004) Shaping the nuclear action of NF-kappaB. Nat Rev Mol Cell Biol 5:392-401.

Chicheportiche Y, Bourdon PR, Xu H, Hsu YM, Scott H, Hession C, Garcia I, Browning JL (1997) TWEAK, a new secreted ligand in the tumor necrosis factor family that weakly induces apoptosis. J Biol Chem 272:32401-32410.

Chicheportiche Y, Chicheportiche R, Sizing I, Thompson J, Benjamin CB, Ambrose C, Dayer JM (2002) Proinflammatory activity of TWEAK on human dermal fibroblasts and synoviocytes: blocking and enhancing effects of anti-TWEAK monoclonal antibodies. Arthritis Res 4:126-133.

Donohue PJ, Richards CM, Brown SA, Hanscom HN, Buschman J, Thangada S, Hla T, Williams MS, Winkles JA (2003) TWEAK is an endothelial cell growth and chemotactic factor that also potentiates FGF-2 and VEGF-A mitogenic activity. Arterioscler Thromb Vasc Biol 23:594-600.

Garcia JH, Lossinsky AS, Kauffman FC, Conger KA (1978) Neuronal ischemic injury: light microscopy, ultrastructure and biochemistry. Acta Neuropathol (Berl) 43:85-95.

Han S, Yoon K, Lee K, Kim K, Jang H, Lee NK, Hwang K, Young LS (2003) 
TNF-related weak inducer of apoptosis receptor, a TNF receptor superfamily member, activates NF-kappa B through TNF receptor-associated factors. Biochem Biophys Res Commun 305:789-796.

Harada N, Nakayama M, Nakano H, Fukuchi Y, Yagita H, Okumura K (2002) Pro-inflammatory effect of TWEAK/Fn14 interaction on human umbilical vein endothelial cells. Biochem Biophys Res Commun 299:488-493.

Hawkins CP, Mackenzie F, Tofts P, du Boulay EP, McDonald WI (1991) Patterns of blood-brain barrier breakdown in inflammatory demyelination. Brain 114:801-810.

Ho DH, Vu H, Brown SA, Donohue PJ, Hanscom HN, Winkles JA (2004) Soluble tumor necrosis factor-like weak inducer of apoptosis overexpression in HEK293 cells promotes tumor growth and angiogenesis in athymic nude mice. Cancer Res 64:8968-8972.

Jin L, Nakao A, Nakayama M, Yamaguchi N, Kojima Y, Nakano N, Tsuboi R, Okumura K, Yagita H, Ogawa H (2004) Induction of RANTES by TWEAK/Fn14 interaction in human keratinocytes. J Invest Dermatol 122:1175-1179.

Kaltschmidt C, Kaltschmidt B, Neumann H, Wekerle H, Baeuerle PA (1994) Constitutive NF-kappa B activity in neurons. Mol Cell Biol 14:3981-3992.

Kim SH, Kang YJ, Kim WJ, Woo DK, Lee Y, Kim DI, Park YB, Kwon BS, Park JE, Lee WH (2004) TWEAK can induce pro-inflammatory cytokines and matrix metalloproteinase-9 in macrophages. Circ J 68:396-399.

Leybaert L (2005) Neurobarrier coupling in the brain: a partner of neurovascular and neurometabolic coupling? J Cereb Blood Flow Metab 25:2-16.

Lin A, Karin M (2003) NF-kappaB in cancer: a marked target. Semin Cancer Biol 13:107-114.

Lynch CN, Wang YC, Lund JK, Chen YW, Leal JA, Wiley SR (1999) TWEAK induces angiogenesis and proliferation of endothelial cells. J Biol Chem 274:8455-8459.

Matsumoto T, Ikeda K, Mukaida N, Harada A, Matsumoto Y, Yamashita J, Matsushima K (1997) Prevention of cerebral edema and infarct in cerebral reperfusion injury by an antibody to interleukin-8. Lab Invest $77: 119-125$.

Mayhan WG (2002) Cellular mechanisms by which tumor necrosis factoralpha produces disruption of the blood-brain barrier. Brain Res 927:144-152.

Michaelson JS, Cho S, Browning B, Zheng TS, Lincecum JM, Wang MZ, Hsu YM, Burkly LC (2005) Tweak induces mammary epithelial branching morphogenesis. Oncogene 24:2613-2624.

Nadjar A, Combe C, Laye S, Tridon V, Dantzer R, Amedee T, Parnet P (2003) Nuclear factor kappaB nuclear translocation as a crucial marker of brain response to interleukin-1. A study in rat and interleukin-1 type I deficient mouse. J Neurochem 87:1024-1036.

Nagai N, De Mol M, Lijnen HR, Carmeliet P, Collen D (1999) Role of plasminogen system components in focal cerebral ischemic infarction: a gene targeting and gene transfer study in mice. Circulation 99:2440-2444.

Nonaka M, Chen XH, Pierce JE, Leoni MJ, McIntosh TK, Wolf JA, Smith DH (1999) Prolonged activation of NF-kappaB following traumatic brain injury in rats. J Neurotrauma 16:1023-1034.

Pahl HL (1999) Activators and target genes of Rel/NF-kappaB transcription factors. Oncogene 18:6853-6866.

Paxinos G, Franklin KBJ (2001) The mouse brain in stereotaxic coordinates. San Diego: Academic.

Polek TC, Talpaz M, Darnay BG, Spivak-Kroizman T (2003) TWEAK mediates signal transduction and differentiation of RAW264.7 cells in the absence of Fn14/TweakR. Evidence for a second TWEAK receptor. J Biol Chem 278:32317-32323.

Potrovita I, Zhang W, Burkly L, Hahm K, Lincecum J, Wang MZ, Maurer MH, Rossner M, Schneider A, Schwaninger M (2004) Tumor necrosis factor-like weak inducer of apoptosis-induced neurodegeneration. J Neurosci 24:8237-8244.
Romanic AM, Madri JA (1994) Extracellular matrix-degrading proteinases in the nervous system. Brain Pathol 4:145-156.

Rong Y, Baudry M (1996) Seizure activity results in a rapid induction of nuclear factor-kappa B in adult but not juvenile rat limbic structures. J Neurochem 67:662-668.

Rosenberg GA, Navratil M, Barone F, Feuerstein G (1996) Proteolytic cascade enzymes increase in focal cerebral ischemia in rat. J Cereb Blood Flow Metab 16:360-366.

Rubin LL, Staddon JM (1999) The cell biology of the blood-brain barrier. Annu Rev Neurosci 22:11-28::11-28.

Saas P, Boucraut J, Walker PR, Quiquerez AL, Billot M, Desplat-Jego S, Chicheportiche Y, Dietrich PY (2000) TWEAK stimulation of astrocytes and the proinflammatory consequences. Glia 32:102-107.

Saitoh T, Nakayama M, Nakano H, Yagita H, Yamamoto N, Yamaoka S (2003) TWEAK induces NF-kappaB2 p100 processing and long lasting NF-kappaB activation. J Biol Chem 278:36005-36012.

Schneider A, Martin-Villalba A, Weih F, Vogel J, Wirth T, Schwaninger M (1999) NF-kappaB is activated and promotes cell death in focal cerebral ischemia. Nat Med 5:554-559.

Shishodia S, Aggarwal BB (2004) Nuclear factor-kappaB activation mediates cellular transformation, proliferation, invasion angiogenesis and metastasis of cancer. Cancer Treat Res 119:139-173.

Tran NL, McDonough WS, Donohue PJ, Winkles JA, Berens TJ, Ross KR, Hoelzinger DB, Beaudry C, Coons SW, Berens ME (2003) The human Fn14 receptor gene is up-regulated in migrating glioma cells in vitro and overexpressed in advanced glial tumors. Am J Pathol 162:1313-1321.

Tran NL, McDonough WS, Savitch BA, Sawyer TF, Winkles JA, Berens ME (2005) The tumor necrosis factor-like weak inducer of apoptosis (TWEAK)-fibroblast growth factor-inducible 14 (Fn14) signaling system regulates glioma cell survival via NF-kappaB pathway activation and BCL-XL/BCL-W expression. J Biol Chem 280:3483-3492.

Wiley SR, Cassiano L, Lofton T, Davis-Smith T, Winkles JA, Lindner V, Liu H, Daniel TO, Smith CA, Fanslow WC (2001) A novel TNF receptor family member binds TWEAK and is implicated in angiogenesis. Immunity 15:837-846.

Witt KA, Mark KS, Huber J, Davis TP (2005) Hypoxia-inducible factor and nuclear factor kappa-B activation in blood-brain barrier endothelium under hypoxic/reoxygenation stress. J Neurochem 92:203-214.

$\mathrm{Xu} \mathrm{H}$, Okamoto A, Ichikawa J, Ando T, Tasaka K, Masuyama K, Ogawa H, Yagita H, Okumura K, Nakao A (2004) TWEAK/Fn14 interaction stimulates human bronchial epithelial cells to produce IL-8 and GM-CSF. Biochem Biophys Res Commun 318:422-427.

Xu L, Zhan Y, Wang Y, Feuerstein GZ, Wang X (2002) Recombinant adenoviral expression of dominant negative IkappaBalpha protects brain from cerebral ischemic injury. Biochem Biophys Res Commun 299:14-17.

Yepes M, Sandkvist M, Moore EG, Bugge TH, Strickland DK, Lawrence DA (2003) Tissue-type plasminogen activator induces opening of the bloodbrain barrier via the LDL receptor-related protein. J Clin Invest 112:1533-1540.

Yepes M, Brown SA, Moore EG, Smith EP, Lawrence DA, Winkles JA (2005) A soluble Fn14-Fc decoy receptor reduces infarct volume in a murine model of cerebral ischemia. Am J Pathol 166:511-520.

Yu Z, Zhou D, Bruce-Keller AJ, Kindy MS, Mattson MP (1999) Lack of the p50 subunit of nuclear factor- $\kappa \mathrm{B}$ increases the vulnerability of hippocampal neurons to excitotoxic injury. J Neurosci 19:8856-8865.

Zhang W, Potrovita I, Tarabin V, Herrmann O, Beer V, Weih F, Schneider A, Schwaninger M (2005) Neuronal activation of NF-kappaB contributes to cell death in cerebral ischemia. J Cereb Blood Flow Metab 25:30-40.

Zonta M, Angulo MC, Gobbo S, Rosengarten B, Hossmann KA, Pozzan T, Carmignoto G (2003) Neuron-to-astrocyte signaling is central to the dynamic control of brain microcirculation. Nat Neurosci 6:43-50. 